\title{
Guest Editorial Workshop on Bayesian Econometric Methods
}

\author{
RODNEY STRACHAN \\ Australian National University and Rimini Centre for Economic Analysis*
}

The 3rd RCEA Bayesian workshop was held in Rimini in July, 2009. The workshop opened with a debate, chaired by Gael Martin, between a leading Bayesian econometrician, Christian Robert, and a leading classical econometrician, Russell Davidson, on the relative virtues of the Bayesian and classical (or frequentist) approaches. The pleasantly civil debate was conducted under the topic "The 21st Century Belongs to Bayes".

The workshop also brought together a variety of both classical and Bayesian econometricians and statisticians, with a view to participants exchanging information on developments in their specific fields of research. The two papers of this volume - one classical in approach and one Bayesian, with insights into classical approaches - partially reflect this purpose, with many more papers having been presented at the workshop itself.

In his paper, Russell Davidson investigates issues in bootstrap testing using, as an example, testing for a unit root in an autoregressive moving average (ARMA) (1,1) process. He focuses on the situation when the root of the MA polynomial is close to minus one. Size distortions in the bootstrap tests result when testing in this situation, due to the near cancellation of the unit AR root with the MA root. Davidson proposes estimators based on nonlinear least squares that are faster to compute than, but not quite as efficient as, the maximum likelihood estimator. These estimators are slower to compute than those proposed by Galbraith and Zinde-Walsh (1994) and (1997), but far more efficient than the Galbraith and Zinde-Walsh estimators. Further, Davidson proposes a new bootstrap procedure that is computationally less demanding than the double bootstrap of Beran (1988). Davidson produces all of his results without recourse to asymptotic theory or asymptotic refinements of the testing procedure.

Polasek, Sellner and Llano consider the problem of estimation and prediction in spatial models when data measurements are taken at different degrees of aggregation and where some observations are missing at one or more levels of aggregation. They adapt the procedure of Chow and Lin (1971), which was developed for time series data with observations on

(C) 2010 Rodney Strachan. Licensed under the Creative Commons Attribution - Noncommercial 3.0 Licence (http://creativecommons.org/licenses/by-nc/3.0/. Available at http: //rofea.org. 
variables at different frequencies, to a model for cross sectional data with spatial autocorrelation. For the parameters in this model they present a maximum likelihood (ML) estimator, which they prove to be best linear unbiased (BLU), and a Bayesian Markov chain Monte Carlo (MCMC) estimator. The authors apply these estimators to interpolate Spanish regional GDP using data measured at two levels of spatial aggregation. In this application, the disaggregated data are fully known and so the authors evaluate the predictive performance of the models with and without the spatial component, and based on both the Bayesian and ML estimators. They find that a Bayesian estimator for a model that accounts for the spatial nature of the data performs best, followed closely by the ML estimator for this spatial model.

RCEA thanks the authors, the referees and Gael Martin for their contributions to the issue and for the dedicated and professional manner in which they have brought this issue to completion. I would like to add my personal thanks to Gael Martin for her tireless work editing the debate. 